\title{
Incarcerated Amyand's Hernia With Acute Appendicitis: A Case Report
}

\author{
Karleigh R. Curfman ${ }^{\mathrm{a}}$, Brendan R. Gontarz ${ }^{\mathrm{a}}$, Michael D. Facciolo ${ }^{\mathrm{a}}$, Meera Cheerharan ${ }^{\mathrm{b}}$, \\ R. Jonathan Robitsek ${ }^{\mathrm{c}}$, Sebastian D. Schuble, d
}

\begin{abstract}
Amyand's hernia is a rare condition defined as the inclusion of the appendix in an inguinal hernia sac, which becomes even rarer in a concomitant case of acute appendicitis. In patients presenting with an incarcerated inguinal hernia and underlying acute appendicitis, the obvious symptoms of the incarceration can mask those of appendicitis. This can complicate management, often leading to a missed pre-operative diagnosis and an emergent intraoperative finding often requiring immediate repair. For this study, we present one case of Amyand's hernia that was diagnosed intraoperatively. The patient is a 58-year-old African American male who presented to his primary care physician for worsening groin pain, later to undergo surgery for an incarcerated hernia. As described, in instances of Amyand's hernia, signs of acute appendicitis may not be initially recognized as they are overshadowed by apparent signs of bowel obstruction. This was the case while our patient was in surgery, as signs suggestive of acute appendicitis were discovered and the patient received an appendectomy in addition to hernia repair. The diagnosis of Amyand's hernia with acute appendicitis has been reviewed in other publications and was found to be a rare condition. As the occurrence rate is so low, there are different methods of management for specific classifications of Amyand's hernia. The case report, background research, statistics, classifications, and specific managements are being presented within this article. The purpose of this study is to evaluate our case against the published data and the procedural recommendations.
\end{abstract}

Keywords: Amyand's hernia; Inguinal hernia; Appendicitis; Appendix

Manuscript accepted for publication September 07, 2015

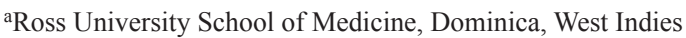

bDepartment of Surgery, Weill Cornell Medical College, New York, NY 10065, USA

'Department of Surgery, Jamaica Hospital Medical Center, Jamaica, NY 11418, USA

${ }^{\mathrm{d} C}$ Corresponding Author: Sebastian D. Schubl, Department of Trauma Surgery, Jamaica Hospital Medical Center, 8900 Van Wyck Expressway, Jamaica, NY 11418, USA. Email: sschubl@jhmc.org

doi: http://dx.doi.org/10.14740/jmc2284w

\section{Introduction}

Amyand's hernia is an eponymous term used for a specific condition in which an inguinal hernia sac contains the vermiform appendix [1]. It derives its name from the French-born English surgeon Claudius Amyand who described the condition during history's first recorded successful appendectomy in 1735, which was performed on an 11-year-old boy with a perforated appendix in the right inguinal hernia sac [2]. The condition is found in $0.5-1 \%$ of all cases of inguinal hernia and $0.07-0.13 \%$ of all cases of appendicitis [2]. Amyand's hernia with appendicitis occurs with four subtypes as follows: type 1 involves a normal appendix within an inguinal hernia, type 2 involves acute appendicitis without inflammation, type 3 involves acute appendicitis with abdominal wall or peritoneal inflammation, and type 4 involves appendicitis with related or unrelated abdominal pathology $[1,3]$.

Patients with Amyand's hernia most typically present with tender right inguinal swelling. Patients usually present with other signs and symptoms of complicated inguinal hernia; however, the incidence is so low that it is rarely diagnosed clinically [4]. In a limited number of instances, it had been diagnosed by computed tomography or ultrasonography preoperatively [5]. An Amyand's hernia is most often discovered and diagnosed intraoperatively [6].

Herein we report a case of type 3 Amyand's hernia occurring in an adult male who presented with tender right inguinal swelling.

\section{Case Report}

A 58-year-old African American male presented to his primary care physician complaining of a bulge in his right groin for the past month, which had become painful 7 days prior to presentation, drastically worsening over the last 2 days. Physical examination revealed a large bulge in the right groin described as irreducible, warm, and moderately tender. Due to the presenting symptoms and physical examination, the patient was sent to the emergency room for surgical evaluation. Routine blood test analysis and a kidney, ureter, and bladder (KUB) Xray (Fig. 1) were performed, showing no abnormalities. After examination by the surgical team and evaluation of laboratory results and imaging, a diagnosis of incarcerated inguinal her- 


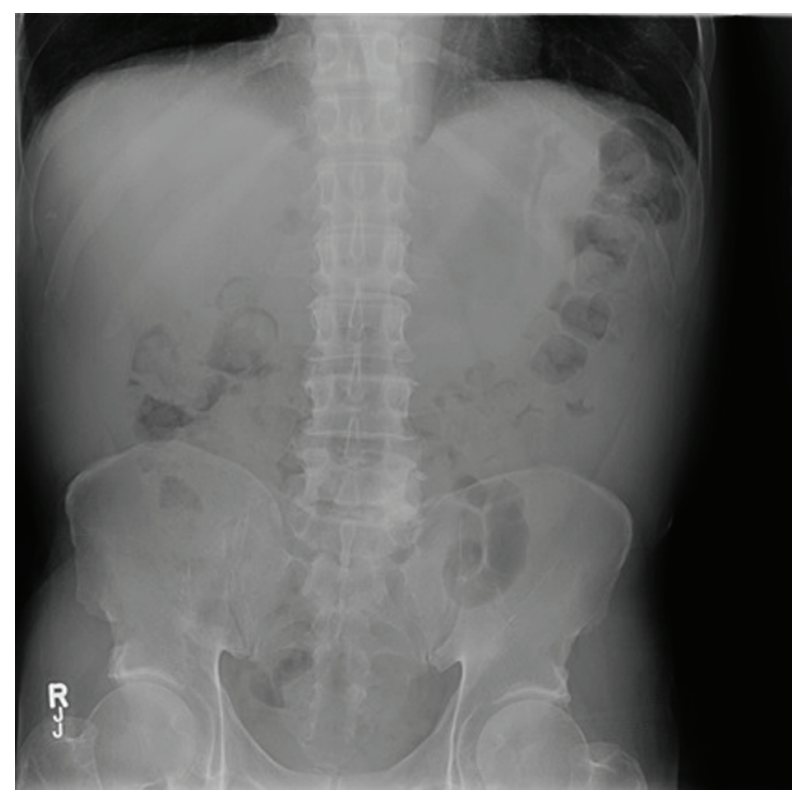

Figure 1. Preoperative kidney, ureter, and bladder X-ray indicated to rule out small bowel obstruction caused by hernia. No dilated bowel loops and a moderate amount of air can be seen, suggestive of nonobstructive bowel gas pattern.

nia was made, and the patient was taken to the operating room for emergency repair.

Intraoperatively, an inflamed, incarcerated appendix and mesoappendix were discovered and found to be fibrotic and adhered within the hernia sac, categorized as type 3 Amyand's hernia (Fig. 2). An appendectomy was then performed due to the intraoperative findings, and the hernia sac was excised. The specimen was sent to pathology for evaluation. The tissue of the hernia was originally intended to be repaired with mesh; however, due to finding of acute appendicitis with inflamma-

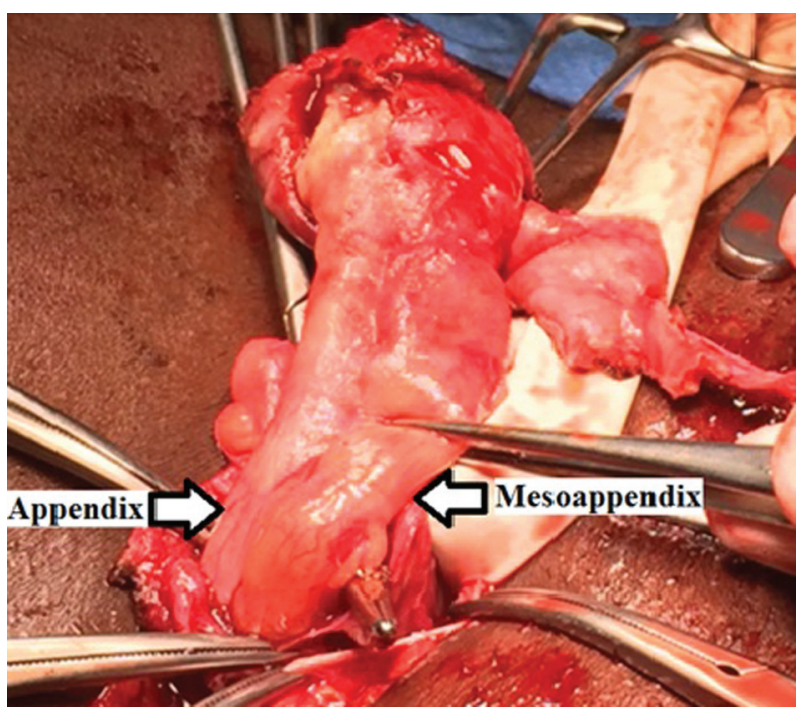

Figure 2. Intraoperatively signs were suggestive of acute appendicitis within the inguinal hernia sac. An inflamed appendix and mesoappendix could be seen once the hernia sac had been dissected.
Table 1. Hernia Types Reported That Included Vermiform Appendix, With the Number of Reported Cases for Each Hernia Type. Adapted From [8]

\begin{tabular}{ll}
\hline Hernia type & Number of cases \\
\hline Incarcerated inguinal hernia & 113 \\
Strangulated inguinal hernia & 11 \\
Incarcerated femoral hernia & 30 \\
Strangulated femoral hernia & 11 \\
Umbilical hernia & 4 \\
Strangulated obturator hernia & 3 \\
Incarcerated Spiegel hernia & 3 \\
Strangulated diaphragmatic hernia & 7 \\
Incisional hernia & 2 \\
Epigastric hernia & 2 \\
Strangulated internal hernia & 1 \\
Strangulated paradouodenoal hernia & 1 \\
Total & 188 \\
\hline
\end{tabular}

tion, placement of mesh was deferred, and a modified Shouldice repair was performed.

The patient was received from the operating room into the unit in stable condition with appropriate vital signs and laboratory results. The patient was discharged home on postoperative day 1 in good condition with no abnormalities or complaints.

\section{Discussion}

Acute appendicitis presenting within a herniated sac is a rare variant accounting for approximately $0.1 \%$ of hernia cases [2]. Inflammation of the appendix is most likely a result of external compression and eventual luminal obstruction from the narrow defect into which the appendix has herniated. The subsequent compromise of blood flow results in further inflammation and bacterial overgrowth [7]. In the existing literature, there have been 188 cases reported of an appendix contained within a hernia sac in 12 varying positions (Table 1) [8].

The diagnosis of Amyand's hernia is difficult to make preoperatively. Though CT scans can suggest appendix involvement in the hernia sac, the diagnosis is typically made intraoperatively. The management, once definitively diagnosed, varies depending on the presentation of the appendix (Table 2) [1]. When presented with a non-inflamed appendix, type 1 , an appendectomy may not be warranted and a Lichtenstein hernia repair remains a viable option. An appendectomy would change the case from a clean to a clean contaminated procedure and raise the risk of infection. Also, visualization of the appendix would require enlarging the hernia defect, therefore increasing the possibility of recurrence [9]. In cases of acute appendicitis, types 2-4, appendectomy is generally indicated.

In repairing the hernia defect, many surgeons argue that use of a mesh or any prosthetic material in a site of established infection increases incidence of infections [10]. The use of a 
Table 2. Classification, description, and suggested management of Amyand's Hernia (Adapted From Losanoff and Basson [1])

\begin{tabular}{lll}
\hline Classification & Description & Surgical management \\
\hline Type 1 & Normal appendix within inguinal hernia & $\begin{array}{l}\text { Hernia reduction, mesh repair, appendectomy } \\
\text { in young patients }\end{array}$ \\
Type 2 & $\begin{array}{l}\text { Acute appendicitis within an inguinal hernia, no abdominal } \\
\text { sepsis }\end{array}$ & $\begin{array}{l}\text { Appendectomy through hernia, primary endogenous } \\
\text { repair of hernia, no mesh }\end{array}$ \\
Type 3 & $\begin{array}{l}\text { Acute appendicitis within an inguinal hernia, abdominal wall } \\
\text { or peritoneal sepsis }\end{array}$ & $\begin{array}{l}\text { Laparotomy, appendectomy, primary repair of hernia, } \\
\text { no mesh }\end{array}$ \\
Type 4 & $\begin{array}{l}\text { Acute appendicitis within an inguinal hernia, related or unrelated } \\
\text { abdominal pathology }\end{array}$ & $\begin{array}{l}\text { Manage as types 1 to 3, investigate or treat second } \\
\text { pathology as appropriate }\end{array}$ \\
\hline
\end{tabular}

Table 3. Surgical Pathology Report From Intraoperative Appendix Tissue Sample Revealing Acute Appendicitis

\begin{tabular}{ll}
\hline Tissue submitted & Description \\
\hline Appendix & Received in formalin fixative and labeled appendix. It consists of an appendix measuring approximately $8.0 \mathrm{~cm}$ \\
& in length and from 1.0 to $2.0 \mathrm{~cm}$ in diameter. The serosal surface is gray/tan and shows patchy areas with tan $/$ gray \\
& fibrinous exudates. The distal end shows a shaggy irregular appearance. There is attached mesoappendix and at \\
& the distal end, an attached portion of fibromembranous-like tissue measuring approximately $5.0 \times 3.0 \times 0.3 \mathrm{~cm}$. \\
& On sectioning, the lumen measures from 0.2 to $0.5 \mathrm{~cm}$. and contains dark brown fecal material. Also received in \\
& the same container is a separate portion of similar appearing fibrous-like tissue measuring $3.0 \times 1.0 \times 0.3 \mathrm{~cm}$. \\
& Final diagnosis: \\
& Appendix, appendectomy: \\
& Acute transmural appendicitis and periappendicitis \\
\hline
\end{tabular}

Shouldice or Bassini technique eliminates the need for mesh and is a viable option in the setting of appendicitis [7, 9].

In this case, the diagnosis of type 3 Amyand's hernia was made intraoperatively with subsequent appendectomy due to appendicitis. The specimen was sent to pathology where a diagnosis of acute appendicitis was made confirming the intraoperative findings (Table 3 ). The defect was repaired via modified Shouldice technique. The management of the appendicitis and the hernia repair were both done in concordance with what current literature describes as the most accepted means of management. The patient was recovering appropriately on postoperative day 1 and was discharged without complications.

\section{Conclusion}

In conclusion, Amyand's hernia is a rare condition consisting of the appendix contained within an inguinal hernia sac. It is rarely diagnosed preoperatively due to its diverse presentation. Amyand's hernia, when discovered intraoperatively, can pose a surgical dilemma. The definitive management is surgical and includes hernia repair with or without appendectomy based on the intraoperative findings [10]. Our case consisted of a patient with a type 3 Amyand's hernia who underwent both hernia repair and appendectomy, consistent with current evidencebased guidelines.

\section{Disclosure}

This case report did not receive any funding support from any organization. The authors declare that there are no conflicts of interest regarding the publication of this paper.

\section{Author Contributions}

Contributors to conception and design, acquisition of data, and interpretation of data: KRC, BRG, MDF, MC. Manuscript writing and drafting: KRC, BRG, MDF, SDS. Revising it critically for important intellectual content: KRC, RJR, SDS. Final approval of the version to be published: KRC, RJR, SDS.

\section{References}

1. Losanoff JE, Basson MD. Amyand hernia: what lies beneath--a proposed classification scheme to determine management. Am Surg. 2007;73(12):1288-1290.

2. Hutchinson R. Amyand's hernia. J R Soc Med. 1993;86(2):104-105.

3. Constantine S. Computed tomography appearances of Amyand hernia. J Comput Assist Tomogr. 2009;33(3):359362.

4. D'Alia C, Lo Schiavo MG, Tonante A, Taranto F, Gagliano E, Bonanno L, Di Giuseppe G, et al. Amyand's hernia: case report and review of the literature. Hernia. 2003;7(2):89-91.

5. Coulier B, Pacary J, Broze B. Sonographic diagnosis of appendicitis within a right inguinal hernia (Amyand's hernia). J Clin Ultrasound. 2006;34(9):454-457.

6. Al-Mayoof AF, Al-Ani BH. Left-sided amyand hernia: 
report of two cases with review of literature. European J Pediatr Surg Rep. 2014;2(1):63-66.

7. Singal R, Gupta S. "Amyand's Hernia" - Pathophysiology, Role of Investigations and Treatment. Maedica (Buchar). 2011;6(4):321-327.

8. Barut I, Tarhan OR. A rare variation of Amyand's Hernia: gangrenous appendicitis in an incarcerated inguinal her- nia sac. Eur J Gen Med. 2008;5:112-114.

9. Mishra VK, Joshi P, Shah JV, Agrawal C, Sharma D, Aggarwal K. Amyand's Hernia: A Case of an Unusual Inguinal Herniace. Indian J Surg. 2013;75(Suppl 1):469-471.

10. Quartey B, Ugochukwu O, Kuehn R, Ospina K. Incarcerated recurrent Amyand's hernia. J Emerg Trauma Shock. 2012;5(4):344-346. 